\title{
Tire-rim interaction, a semi-analytical tire model
}

\author{
F. Ballo · G. Previati - M. Gobbi · G. \\ Mastinu
}

\begin{abstract}
This paper deals with the development and validation of a semi-analytical tire model able to compute the forces at the interface between tire and rim. The knowledge of the forces acting on the rim is of crucial importance for the lightweight design of wheels. The proposed model requires a limited set of data to be calibrated. The model is compared with complete FE models of the tire and rim.

Despite its simplicity, the semi-analytical model is able to predict the forces acting on the rim, in agreement with the forces computed by complete FE models. The stress state in the wheel rim, computed by the developed semi-analytical model matches fairly well the corresponding stress state coming from experimental tests.
\end{abstract}

Keywords tire modeling, ring model, finite element modeling, tire testing

\section{Introduction}

In the design of the wheel rim, one critical point is the knowledge of the force due to the tire-rim interaction. The tire is a very complex system, made from different materials and with a highly non linear behaviour. In such a situation, the accurate knowledge of the transmitted force is a key factor for the lightweight design of the rim and of the whole wheel.

Several studies can be found in the literature on the simulation and prediction of the contact forces generated at tire-road interface (see for instance the books $[1,2])$. In few papers, however, the tire rim-interaction is investigated. In many cases, the load acting on the tire are roughly estimated by simplified distributions [3-7] at the rim.

Finite element models are often employed to realize high accurate physical models of the tire $[8,9]$. Such models are very informative and able to predict the tire-rim interaction forces. However, the computational cost, the development

Politecnico di Milano,

via La Masa 1 ,

20156, Milano

email: federicomaria.ballo@polimi.it 
time and the large amount of required information on the structure of the tire [10-15] could make these models not suitable to be used, in case the focus of the designer is on the wheel rim.

Simplified structural models of tires can be found in some papers. In [1618], the radial stiffness of the tire is computed from geometrical parameters of the tire and the inflating pressure. These models are able to give a fairly good approximation of the load carrying characteristics of the pneumatic tire.

Several papers can be found on the in-plane dynamics of the tire, described by rigid rings tire models $[19,20]$. These models are mostly used for the simulation of vertical and longitudinal mechanical behaviour of the tire. In [21-23] the tire is considered a flexible ring allowing the study of the vehicle handling and low frequency comfort. The tire is modelled as deformable ring laying on a elastic foundation representing the (linearised) elastic response of the tire sidewalls and of the inflating pressure. The same approach is followed in $[24,25]$ to compute the interaction forces between a motorcycle tire and the rim. In the paper the above mentioned tire models are somehow combined to derive a proper tire model to estimate forces at tire-rim interface.

In this paper a semi-analytical model of a car tire for the computation of the tire-rim interaction forces is proposed. In the first section, a semi-analytical mathematical tire model is presented. The model includes a simplified geometrical description of the tire cross-section deformation, the load carrying mechanism of the sidewalls of the tire and a deformable ring model of the tire. Then, the (few) model parameters are experimentally identified with a very simple experimental procedure. Finally, a validation of the model is shown considering FE models of both the tire and the wheel. The output of the models are further validated by comparing the computed strain at the wheel and the corresponding strains measured by means of strain gauges.

\section{Semi-analytical model of the tire}

In this section the semi-analytical model of the tire is described. Firstly, the tire geometry as function of the vertical deformation is computed. Then, the load carrying and stiffness properties of the tire in the radial direction can be computed by considering two different phenomena acting concurrently, namely the effect of the inflating pressure and the structural stiffness of the carcass. Finally, the semianalytical model of the radial deformation of the tire as a curved beam is presented.

\subsection{Geometrical description of a deformed cross-section of the tire}

The tire can be considered divided into parts having different structural behaviour (Fig. 1). Such parts are the tread belt and the two sidewalls. The tread belt is very stiff in the circumferential direction and can be considered of constant length. Moreover, the tread belt has a high bending stiffness along the circumferential axis. The geometric dimensions of a tire cross section are shown in Fig. $1 . b_{c}$ represents the width of the contact area.

The sidewalls of the tire have a small stiffness and in the very first approximation can be considered as membrane. Under such of a hypothesis, the sidewalls 


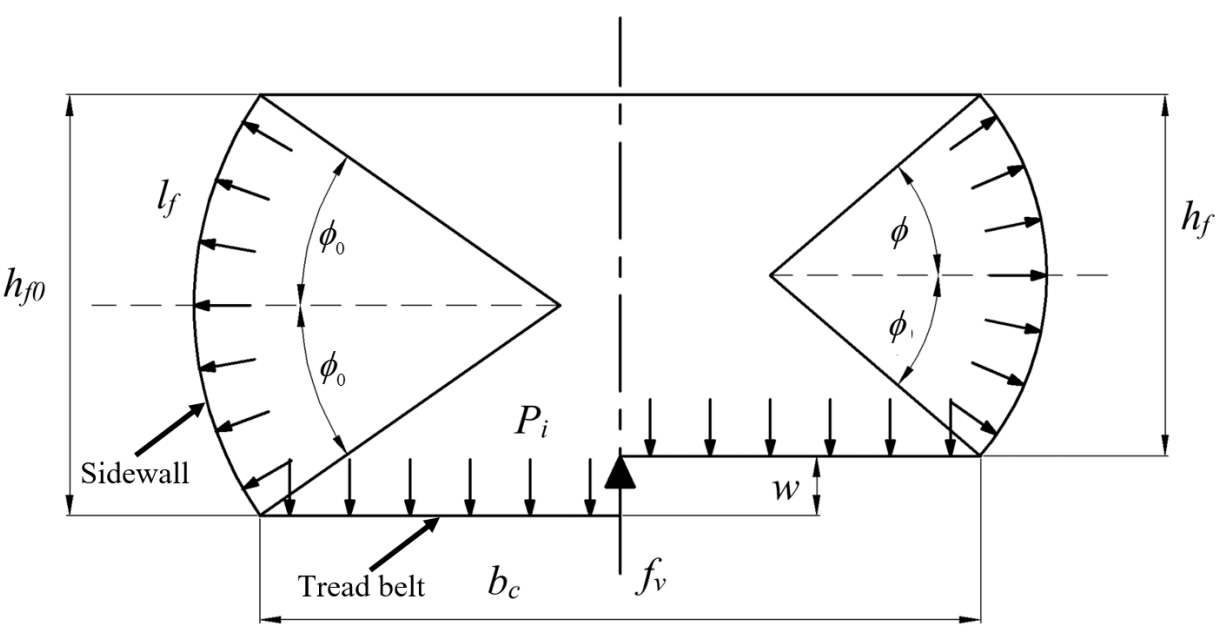

Fig. 1 Cross section of a pneumatic tire. Left: undeformed section. Right: deformed section when a force $f_{v}$ is applied.

of the tire, under the action of the internal pressure $P_{i}$, assume a circular shape. The centre of circumference has a lateral displacement with respect to the median plane of the section [17], as a vertical displacement $w$ is applied (Fig. 1 right). During the deformation, both the length of the $\operatorname{arc} l_{f}$ and the contact width $b_{c}$ remain constant. The height of the section reduces from the undeformed height $h_{f 0}$ to the deformed height $h_{f}$.

Considering Fig. 1 right, the following relationship between the vertical displacement $w$ and the angle $\phi$ can be obtained.

$$
w=h_{f 0}-l_{f} \frac{\sin (\phi)}{\phi}
$$

In the neighbourhood of a given angle $\bar{\phi}, \sin (\phi)$ can be linearised as

$$
\sin (\phi) \simeq \sin (\bar{\phi})+\cos (\bar{\phi})(\phi-\bar{\phi})
$$

By replacing eq. 2 in eq. 1, the expressions of $\phi=\phi(w)$ can be derived and reads

$$
\phi \simeq \frac{A_{1}}{w+A_{2}}
$$

where

$$
\begin{array}{r}
A_{1}=l_{f}(\bar{\phi} \cos (\bar{\phi})-\sin (\bar{\phi})) \\
A_{2}=l_{f} \cos (\bar{\phi})-h_{f 0}
\end{array}
$$

The value of $\bar{\phi}$ for each given value of the vertical deformation $\bar{w}$ can be easily found by numerically solving eq 1 . 


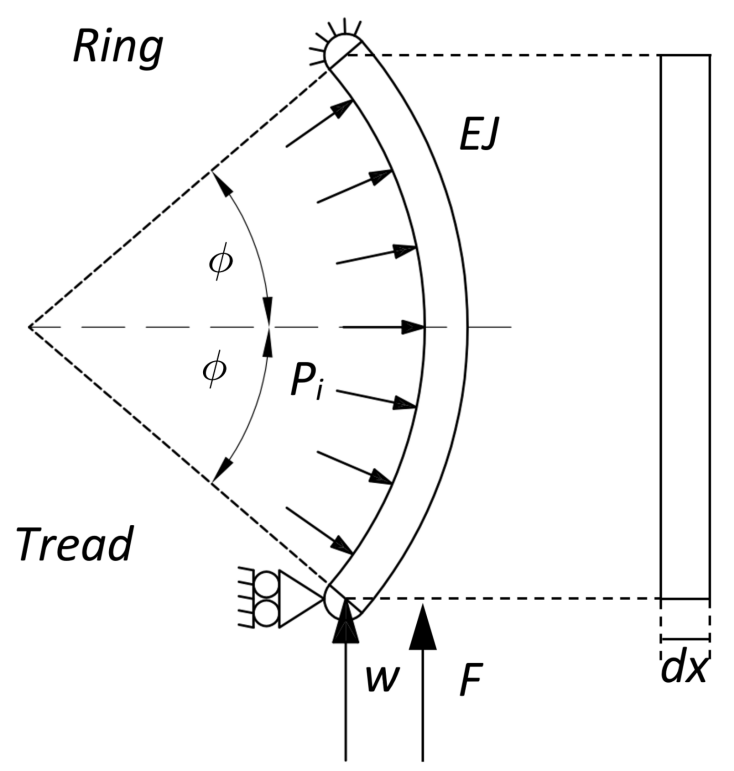

Fig. 2 Infinitesimal section of the sidewall of the tire as a curved beam.

\subsection{Load carrying mechanism of the sidewall of the tire}

In the previous subsection, the sidewalls of the tire are considered as membranes without any structural stiffness. On the contrary, each sidewall of the tire can be modelled as a curved beam, of small but not negligible bending stiffness, under the combined action of the internal pressure and of a vertical displacement. The beam reacts with a force opposite to the displacement (Fig. 2). The beam is pinned to the rim and tread belt.

By considering the conventions of Fig. 2, the displacement $w$ can be computed as $[26]$

$$
w=\frac{R^{3}\left(1-\nu^{2}\right)}{E J} F \cdot K_{c}+\frac{R^{4}\left(1-\nu^{2}\right)}{E J} P_{i} \cdot K_{d}
$$

where $R$ is the radius of curvature of the beam and the term $1-\nu^{2}$ considers the transversal constraint of the beam (given by the sidewall), $\nu$ is the Poisson coefficient of the material. If the beam is thin and the hoop-stress is neglected, the two load factors $K_{c}$ and $K_{d}$ read [26]

$$
\begin{array}{r}
K_{c}=\phi \cos (\phi)^{2}+\phi \sin (\phi) \cos (\phi)+\phi-3 \sin (\phi) \cos (\phi) \\
K_{d}=\cos (\phi)\left(\phi \sin (\phi)^{2}-2 \phi+3 \sin (\phi) \cos (\phi)\right)
\end{array}
$$

From eq. 6 the force $F$ can be computed

$$
F=\frac{E J}{R^{3}\left(1-\nu^{2}\right) K_{c}} w+K_{d c} R P_{i}, \text { with } K_{d c}=-\frac{K_{d}}{K_{c}}
$$




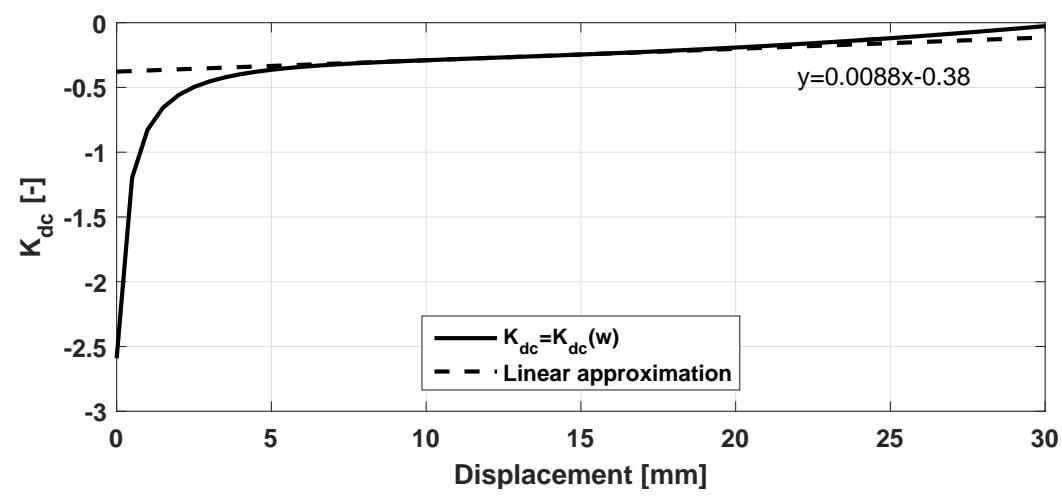

Fig. 3 Expression of $K_{d c}=K_{d c}(w)$ as function of $w$ (data in table 2).

It can be observed that since $R=R(w)$ and $K_{d c}=K_{d c}(w)$, the value of the force $F$ depends on the actual deformation of the tire. The expression of $R=R(w)$ can be derived from Fig. 1 and, by considering eq. 3, reads

$$
R=\frac{l_{f}}{2 \phi} \simeq \frac{l_{f}\left(A_{2}+w\right)}{2 A_{1}}=\bar{R}+\frac{l_{f}(w-\bar{w})}{2 A_{1}}
$$

where $\bar{R}$ is the value of the radius when the $\phi=\bar{\phi}$, the expressions of $A_{1}$ and $A_{2}$ are reported in eq. 4 and eq. 5 respectively.

The expression of $K_{d c}=K_{d c}(w)$ is highly non linear with $w$. In Fig. $3 K_{d c}$ as function of $w$ is depicted (data in table 2). In the region of interest, from 5 to $25 \mathrm{~mm}$ of displacement, the curve is fairly linear and can be interpolated by a straight line. In the following, a linearised expression

$$
K_{d c} \simeq K_{d c 1} w+K_{d c 0}
$$

will be considered.

By replacing eq. 10 and eq. 11 in eq. 9 and dropping all the non linear terms, the expression of the force $F$ can be approximated as

$$
F=P_{i} B_{1}+B_{2} w+P_{i} B_{3} w
$$

where

$$
\begin{array}{r}
B_{1}=K_{d c 0} \bar{R} \\
B_{2}=\frac{E J}{\bar{R}^{3}\left(1-\nu^{2}\right) \bar{K}_{c}} \\
B_{3}=K_{d c 1} \bar{R}+K_{d c 0} \frac{l_{f}}{2 A_{1}}
\end{array}
$$

where $\bar{K}_{c}$ is the value of $K_{c}$ at $w=\bar{w}$.

Eq. 12 states that the load carrying mechanism of the sidewalls of the tire can be approximated as a linear spring with stiffness given by a constant term function of the structure of the sidewalls and a term proportional to the inflating pressure 
representing the stiffening effect of the pressure. The term $P_{i} B_{1}$ represents the effect of the pressure that acts to reduce the radius of curvature of the beam and is balanced by the pressure acting on the tread.

\subsection{Tyre model}

The analytical model describing the radial deformation of the tire is derived from the model described in Kim et al. in [21] and in Ballo et al. [25]. The model was originally derived in [21] to compute pressure and shear stress/strain distributions in the contact patch of a car tire under a vertical load. In [25], it has been adapted to the computation of the tire/rim interface forces for the radial deflection of a motorcycle tire.

In the following, the model firstly presented in [21] and modified in [25] is briefly summarized.

The model is depicted in Fig. 4 and has been derived under the following hypothesis.

- The tread belt is described by a deformable curved elastic beam under small deformations with moment of inertia $J$ and the material Young modulus $E$.

- The tread belt is connected to the rim by means of a distributed linear spring $k$ representing the residual radial stiffness per unit of length of the tire carcass.

- The rim is described by the internal rigid ring of Fig. 4 fixed to the ground.

- The tire is loaded in the radial direction and the model is symmetric with respect to a vertical axis passing through the centre of the tire.

The residual stiffness per unit of length of the carcass $k$ considers also the stiffening effect of the inflation pressure

$$
k=k_{0}+k_{1} \cdot P_{i}
$$

where, according to the discussion of Section 2.1 and Section 2.2, the two coefficients of eq. 16 can be estimated as $k_{0}=2 B_{2}$ and $k_{1}=2 B_{3}$ (two sidewalls are considered).

Fig. 5 depicts the kinematics of a portion $d s$ of the tread defined by the infinitesimal angle $d \xi$.

By considering a positive displacement $u$ in the radial direction $n$ that moves the point $Q$ to $Q_{d}$ and a radial vector $r$ pointing towards the centre, under the condition $u \ll r$, the following kinematic quantities can be computed [25].

- Length $d s_{d}$ of the deformed arch

$$
d s_{d}=d s-\frac{u}{r} d s
$$

- Angle $d \xi_{d}$ in the deformed configuration

$$
d \xi_{d}=d \xi-\frac{d^{2} u}{d s^{2}} d s
$$




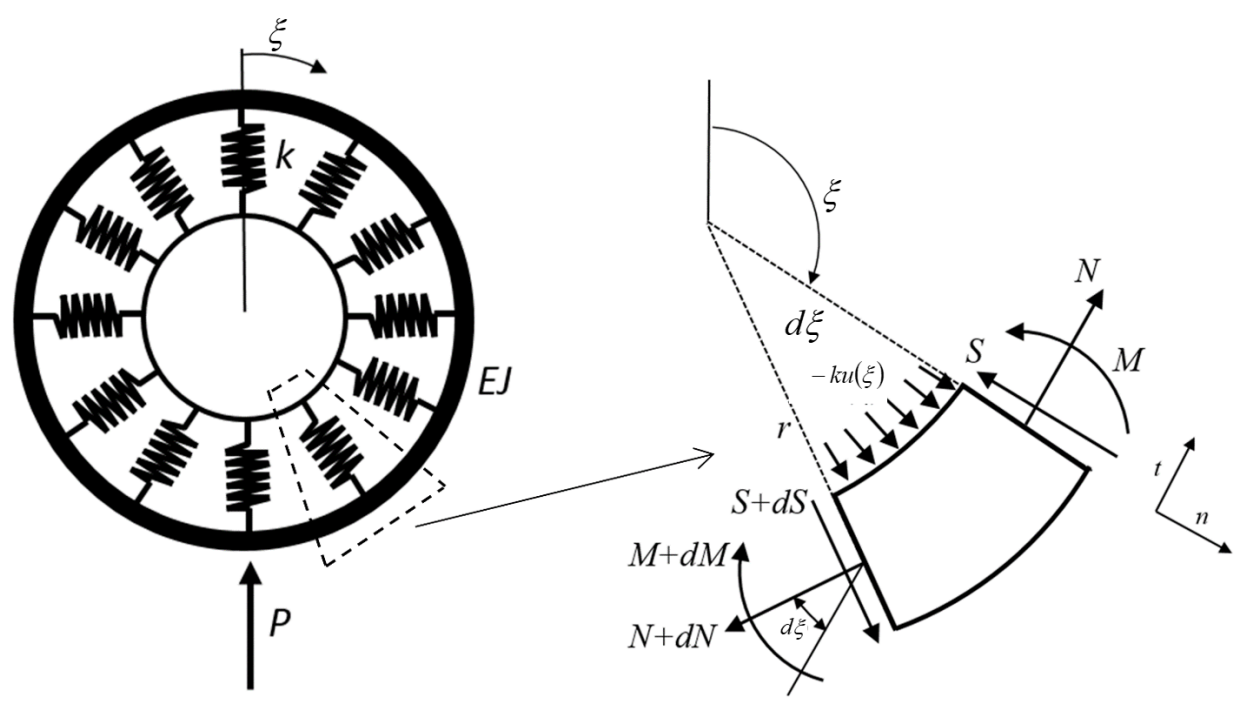

Fig. 4 Simplified analytical tire model for radial deflection [21]. Left: tread belt modeled as a curved beam connected to the rim (fixed to the ground) by springs. Right: Free body diagram of a portion $d s$ of the curved beam representing the tread belt.

- Curvature in the undeformed configuration

$$
\frac{1}{r}=-\frac{d \xi}{d s}
$$

- Curvature in the deformed configuration

$$
\frac{1}{r_{d}}=-\frac{d \xi_{d}}{d s_{d}} \simeq \frac{1}{r}+\frac{u}{r^{2}}+\frac{d^{2} u}{d s^{2}}
$$

By considering the kinematic quantities in eq. 17-20, the relationship between the change in the curvature and the bending moment

$$
\frac{1}{r_{d}}-\frac{1}{r}=-\frac{M}{E J}
$$

can be rewritten as $[21,25]$

$$
\frac{1}{r^{2}}\left(\frac{d^{2} u}{d \xi^{2}}+u\right)=-\frac{M}{E J}
$$

In Fig. 4 Right the forces acting on a portion $d s$ of the curved beam are depicted. By considering the equilibrium in the radial and tangential directions and with respect to the rotation and by neglecting the higher order terms, the following equations can be derived [25]

$$
\begin{array}{r}
\frac{d S}{d \phi}=N+k r u(\xi) \\
d N=-S d \phi \\
S=\frac{1}{r} \frac{d M}{d \xi}
\end{array}
$$




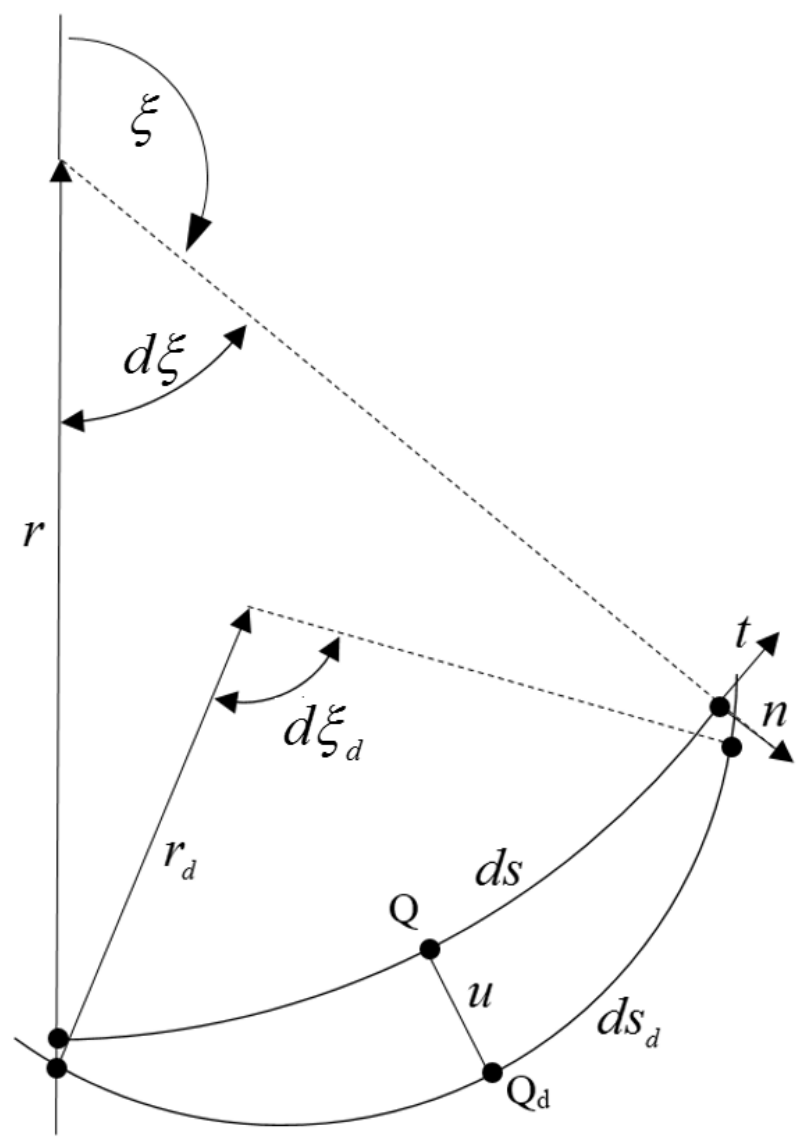

Fig. 5 Portion $d s$ of a general curved beam

Finally, by substituting equation 25 in 23 , deriving with respect to $\xi$ and considering equations 24 and 22 , the differential equation describing the relationship between te radial displacement $u(\xi)$ and the bending moment $M$ can be obtained [25].

$$
\frac{d^{5} u}{d \xi^{5}}+2 \frac{d^{3} u}{d \xi^{3}}+\left(1+\frac{k r^{4}}{E J}\right) \frac{d u}{d \xi}=0
$$

Equation 26 is a homogeneous linear differential equation, with general solution

$$
u(\xi)=U_{1} e^{m_{1} \xi}+U_{2} e^{m_{2} \xi}+U_{3} e^{m_{3} \xi}+U_{4} e^{m_{4} \xi}+U_{5} e^{m_{5} \xi}
$$

by defining $\delta=\sqrt{\frac{k r^{4}}{E J}+1}, \alpha=\sqrt{\frac{\delta-1}{2}}$ and $\beta=\sqrt{\frac{\delta+1}{2}}$, Eq. 27 can be rewritten as [25]

$$
\begin{aligned}
u(\xi)= & C_{0}+\cos (\beta \xi)\left[C_{1} \cosh (\alpha \xi)+C_{2} \sinh (\alpha \xi)\right]+ \\
& \sin (\beta \xi)\left[C_{3} \cosh (\alpha \xi)+C_{4} \sinh (\alpha \xi)\right]
\end{aligned}
$$


where the five constants can be computed by imposing the symmetry conditions $\frac{d u}{d \xi}=0$ both at $\xi=0$ and $\xi=\pi$, the equilibrium of the shear force at $\xi=0$ and $\xi=\pi\left(S_{\xi=0}=0\right.$ and $S_{\xi=\pi}=-P / 2$ respectively $)$ and the conservation of the length of the beam. This last condition can be expressed as $\int_{0}^{\pi}\left(\frac{d^{2} u}{d \xi^{2}}+u\right) d \xi=0$ [25]. The expressions of the coefficients are reported in Appendix A.

A distributed load can be considered simply as a superposition of single concentrated loads. The angular sector of the contact patch can be discretized by considering $N$ increments $\Delta \xi_{i}$. The tire radial deflection can be expressed as

$$
u(\xi)=\sum_{i=1}^{N} P\left(\xi_{i}\right) \Delta \xi_{i} r \bar{u}\left(\xi, \xi_{i}\right)
$$

where $\bar{u}\left(\xi, \xi_{i}\right)$ is the radial deformation of the tire subjected to a concentrated unit load at $\xi_{i} . P\left(\xi_{i}\right) \Delta \xi_{i}$ is the discretized load on the considered interval. In the current paper, a parabolic load will be considered.

The distributed load is applied on the contact patch of the tire. The contact patch $(L)$ is computed by considering the formula proposed in [1] as

$$
L=p_{a 1}\left(p_{a 2} \sqrt{\frac{u}{r}}+\frac{u}{r}\right) r
$$

with $p_{a 1}$ and $p_{a 2}$ interpolation parameters. For a flat surface, the parameters $p_{a 1}$ and $p_{a 2}$ assume the values $p_{a 1}=0.35$ and $p_{a 2}=2.25[1]$.

\subsection{Forces acting on the rim}

After we have derived the tire model, we can focus on the forces acting on the wheel rim. Only the reaction forces between rim and tire in the radial and axial direction are considered. Circumferential forces due to the radial load are neglected as are of little interest in the design of the rim.

In Fig. 6 the forces acting at tire-rim interface on an infinitesimal cross section of the tire are depicted. In the following the forces in radial and axial directions are explained.

The forces (per unit of length) in the radial direction are given by three contributions:

1. Force due to the inflating pressure. In Fig. 7, the sidewall has been modeled as a membrane element $[16,17,27]$ and transmits the tension force $V$ to the rim. The radial component of $V$ reads

$$
T_{\text {rad,p }}=V \cos (\phi)=\frac{p h_{f 0}}{2 \tan (\phi)}
$$

This radial component of the tension force in the sidewall due to the inflating pressure partially acts on the rim and partially on the bead of the tire. In particular, on the bead a circumferential force will arise which limits the radial deformation of the bead itself ( $T_{\text {bead }}$ in Fig. 6$)$. When the bead is stiff, most of the radial force due to the pressure acts on the bead and only a small fraction 
on the rim. The relative distribution of this force depends on the considered tire. For the tire considered, the force acts exclusively on the bead and not on the rim, i.e. on the $\operatorname{rim} T_{\text {rad,p }}=0$.

2. Radial reaction of the rim to the axial force. The axial component of $V$ acts on the upper part of the rim which can be considered an inclined plane. The inclination can be estimated by considering the zone where the maximum pressure is acting as shown in Fig. 8. For the considered rim this inclination is about $25^{\circ}$ and can be estimated by considering the inclination of the line connecting the end point of the fillet between bottom and lateral contact zone of the tire and the separation point between the upper part of the rim flange and the tire. Calling $T_{a x}$ the axial force acting on the rim (computed in the following), the radial projection due to the inclined plane can be computed as

$$
T_{\text {rad, } a}=T_{a x} \tan (\theta)
$$

with $\theta$ the angle of action of the part of the rim in contact with the tire.

3. The additional force (with respect to $T_{\text {rad,p }}$ ) due to the tire deformation caused by loading. The radial force per unit length due to the deformation of the tire acting on the wheel rim can be computed as

$$
T_{\text {rad,q }}=\frac{1}{2} k u(\xi) \frac{2 \pi r}{2 \pi r_{\text {rim }}}
$$

where the scaling factor $\frac{2 \pi r}{2 \pi r_{\text {rim }}}$ accounts for the different values of the radius of the tire and the rim and the coefficient $1 / 2$ for the presence of two sidewalls.

The forces (per unit of length) in the axial direction (i.e. the direction of the wheel rotation axis) have two contributions.

1. Forces due to pressure in the axial direction. Referring to the scheme of Fig. 7, and considering the deformation of the sidewall (i.e. $h=h(\phi)$ ), the axial force can be expressed as

$$
T_{a x, p} \simeq \frac{p h(\xi)}{2}=\frac{p\left(h_{v 0}+u(\xi)\right)}{2}
$$

2. Structural reaction of the sidewall. When deformed radially, the sidewall, given the curved shape, reacts also in the axial direction. The amount of this axial force depends on the stiffness and geometry of the sidewall and on the stiffness of the joint between sidewall and tread. The axial force can be computed as

$$
T_{a x, q}=\frac{k_{a x}}{k} T_{r a d, q}
$$

where $k_{a x}$ is the axial stiffness of the sidewall. To estimate $k_{a x}$, different geometries have been evaluated by a FEM model and values of the axial stiffness between $20 \%$ and $40 \%$ of the radial one have been obtained. For the tire considered in this paper a value $k_{a x}=0.3 k$ is assumed.

The total forces acting on the rim are given by the sum of all the contributions and read

$$
\left\{\begin{array}{l}
T_{r a d}=T_{r a d, a}+T_{r a d, q} \\
T_{a x}=T_{a x, p}+T_{a x, q}
\end{array}\right.
$$




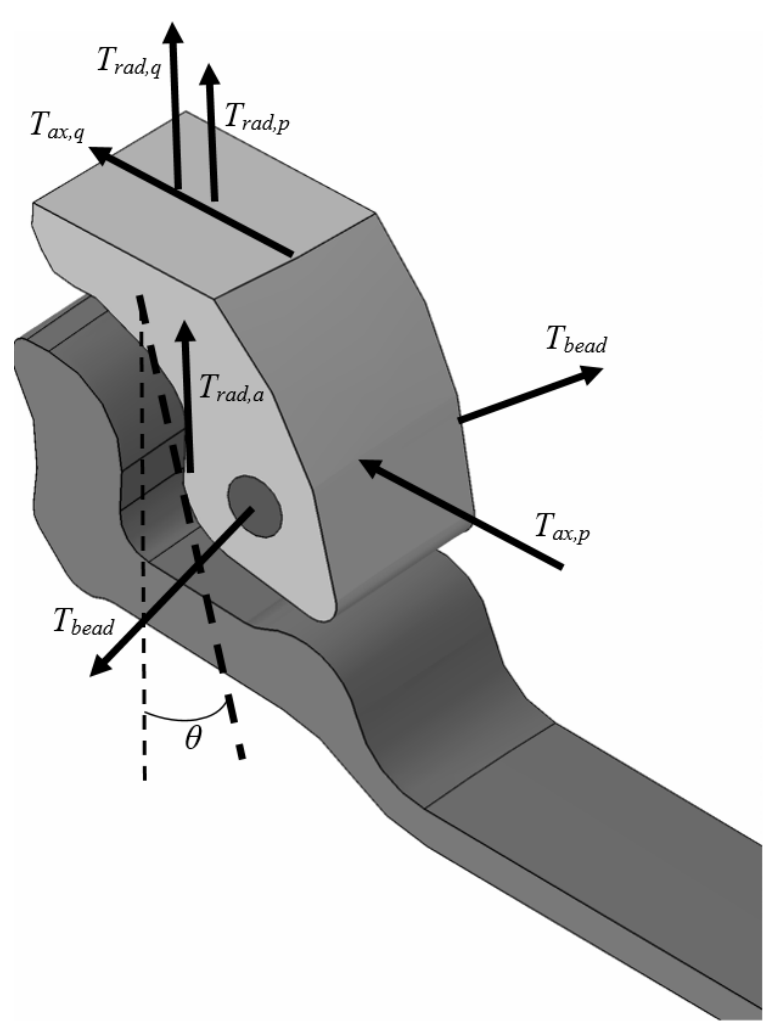

Fig. 6 Forces acting at tire-rim interface on a infinitesimal section of the tire.
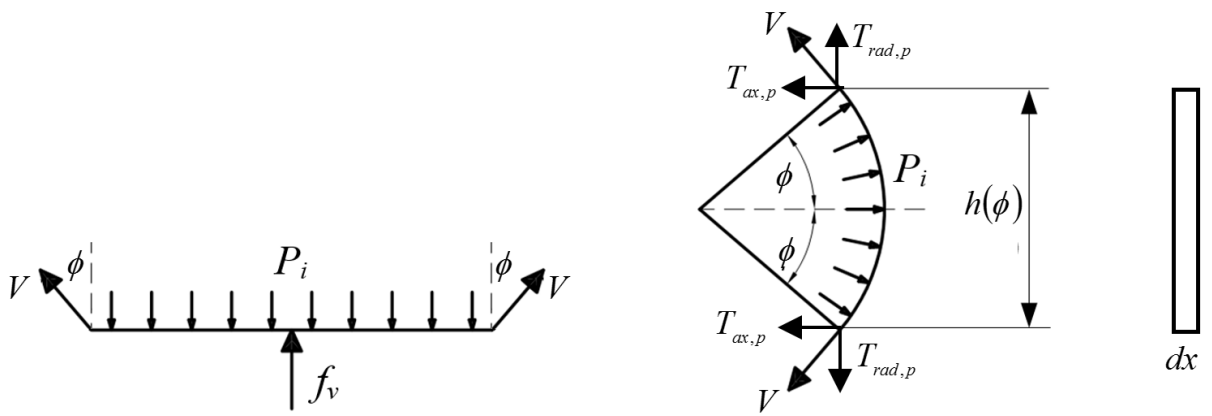

Fig. 7 Forces acting on a section of a pneumatic tire when only the internal pressure is applied.

\section{Experimental tests and parameters identification}

A series of experimental tests have been realized in order to identify the parameters of the analytical model of the tire. In this section, firstly the experimental tests are presented. Then, the procedure for the identification of the parameters is described. 


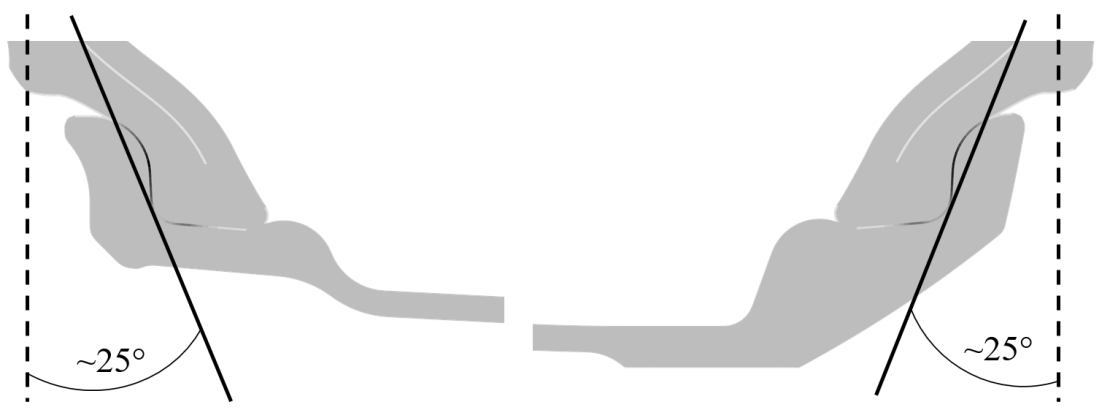

Fig. 8 Estimation of the inclination of the plane of action of the pressure axial force on the rim. The inclination of the plane is defined as the inclination of the line connecting the extreme points of the pressure zone (dark grey zone in the lateral contact area). These points can be estimated as the end point of the fillet between bottom and lateral contact zone of the tire and the separation point between the upper part of the rim flange and the tire.

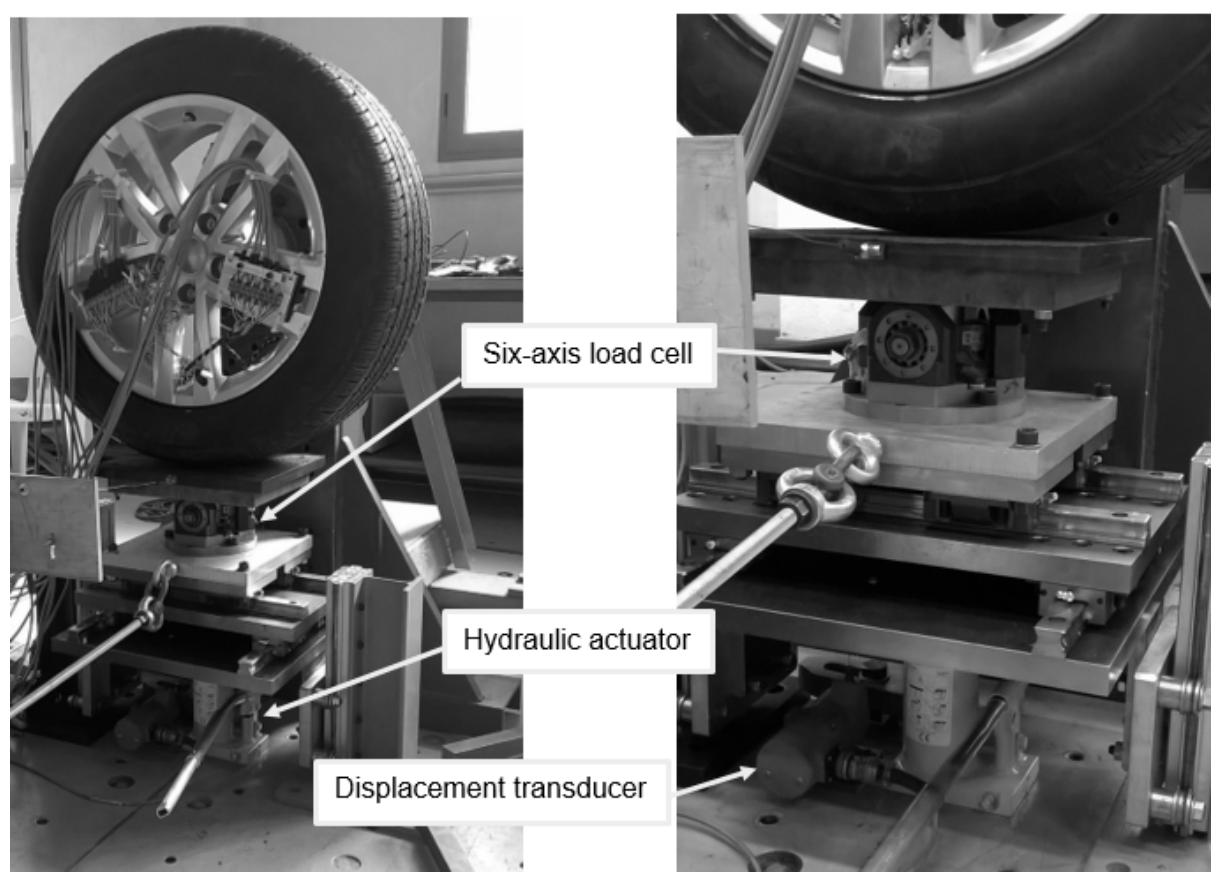

Fig. 9 Test bench for tire stiffness measurement (left) and detail of the moving platform (right)

\subsection{Experimental tests}

For the experimental tests, the specifically designed test bench for measuring the radial stiffness of the tire shown in Fig. 9 has been used. By this test bench, different values of vertical load can be applied and the deformation of the tire can be measured. 
The test bench consists of a hydraulic actuator that provides the vertical load by moving a horizontal platform in the vertical direction. The wheel is fixed to the ground and is deformed by the platform. A six-axis load cell is mounted between the actuator and the platform. The employed load cell has been conceived, realized and tested at Politecnico di Milano. The six-axis force sensor is thoroughly described in several papers $([28-30])$. The sensor performances are reported in Table 1. By using a six axis load cell, the vertical load can be measured without requiring any guiding mechanisms to move the platform. The horizontal platform (see Fig. 9 (right)) allows lateral and longitudinal movements. In this way, when loading the system, unwanted longitudinal and lateral forces can be avoided. The vertical displacement of the platform has been measured by means of the displacement transducer shown in Fig. 9 (right).

Table 1 Technical data of the six-axis load cell

\begin{tabular}{llll}
\hline Maximum force & $F_{x}$ & 10 & $\mathrm{kN}$ \\
& $F_{y}$ & 20 & $\mathrm{kN}$ \\
& $F_{z}$ & 10 & $\mathrm{kN}$ \\
Uncertainty & $95 \%$ Full Scale (F.S.) & 0.35 & $\%$ \\
Maximum torque & $M_{x}$ & 0.5 & $\mathrm{kNm}$ \\
& $M_{y}$ & 0.25 & $\mathrm{kNm}$ \\
Uncertainty & $M_{z}$ \% Full Scale (F.S) & 0.5 & $\mathrm{kNm}$ \\
Total mass & $95 \% 23$ & $\%$ \\
Bandwidth & & 2.7 & $\mathrm{~kg}$ \\
Cross-talk & & 350 & $\mathrm{~Hz}$ \\
Hysteresis & $\%$ F.S. & $< \pm 1 \%$ & \\
\hline
\end{tabular}

The measure of tire under test is $265 / 50 \mathrm{R} 19$. The vertical characteristics have been measured for four different levels of pressure $(2.1,2.5,3$ and 4.5 bar) and for loads from 1500 to $9000 \mathrm{~N}$.

\subsection{Parameters identification}

The structural parameters $k_{0}, k_{1}$ and $E J$ of the analytical model have to be identified. Before the actual identification of the parameters by the experimental tests, an estimation of the values of such parameters can be obtained by considering the formulae in Section 2.2.

The profile of the tire has been measured by a portable coordinate measuring machine (CMM) and is reported in Fig. 10. The main geometrical and mechanical properties of the tire are reported in table 2 . The elastic modulus of the material has been estimated on the basis of the material parameters reported in table 4

In Section 2.3, following the discussion of Section 2.1 and Section 2.2, the values of $k_{0}$ and $k_{1}$ of eq. 16 have been estimated as $k_{0}=2 B_{2}$ and $k_{1}=2 B_{3}$. According to eq. 14 and 15 , the values of $B_{2}$ and $B_{3}$, and hence of $k_{0}$ and $k_{1}$, depend on the deformation of the tire. A reference deformation $\bar{w}=10 \mathrm{~mm}$ is considered. By considering the values of table 2 , from eq. 14 , remembering $k_{0}=2 B_{2}$, the estimated value of $k_{0}$ ranges between $0.005 \mathrm{~N} / \mathrm{mm}^{2}$ if the thickness of the sidewall 


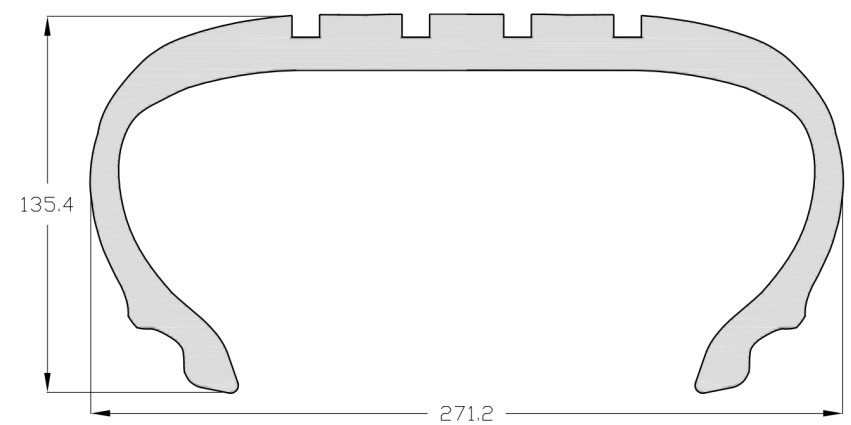

Fig. 10 Measured section of the considered tire

is considered at the lower limit of $8 \mathrm{~mm}$ and $0.018 \mathrm{~N} / \mathrm{mm}^{2}$ if it is considered at the upper limit of $12 \mathrm{~mm}$. The value of $k_{1}=2 B_{3}$ can be estimated from eq. 15 and it is $2.02[-]$.

The value of $E J$ can be roughly estimated by considering the section of the tire as a "C" section with similar dimensions made from a composite material. By this simplification, the order of magnitude of $E J$ can be computed between $10^{8}$ and $10^{9} \mathrm{Nmm}^{2}$.

Fig. 11 depicts the comparison between the experimental and computed values. In table 3 , the identified values of the parameters are shown.

Table 2 Geometrical and mechanical data of the considered tire

\begin{tabular}{ll}
\hline Tyre measure & $265 / 50$ R19 \\
$r[\mathrm{~mm}]$ & 374 \\
$l_{f}[\mathrm{~mm}]$ & 13.5 \\
$h_{f v 0}[\mathrm{~mm}]$ & 103.5 \\
$\bar{w}[\mathrm{~mm}]$ & 10 \\
$\bar{\phi}[\mathrm{deg}]$ & 60.6 \\
$\bar{R}[\mathrm{~mm}]$ & 53.7 \\
$E$ (sidewall) ${ }^{1}[\mathrm{MPa}]$ & 3.42 \\
$\nu$ (sidewall $)[-]^{-}$ & 0.5 \\
\hline
\end{tabular}

\section{Rim forces computation and FEM comparison}

In this section, the forces acting at the rim computed by the semi-analytical model are compared with the same forces computed by a FE model of the considered tire.

${ }^{1}$ For a rubber-like material described by a Mooney-Rivlin hyperlastic model, the relationship between the elastic modulus $E$ and the material parameters $C_{10}$ and $C_{01}$ reads [31] (data in table 4)

$$
E=6\left(C_{10}+C_{01}\right)
$$


Table 3 Semi-analytical model parameters (eqs. 12,16,26): respective ranges for identification and identified values

\begin{tabular}{ll}
\hline Parameter & Range \\
\hline$k_{0}\left[\mathrm{~N} / \mathrm{mm}^{2}\right]$ & $0.005-0.020$ \\
$k_{1}[-]$ & $1-3$ \\
$E J\left[N \cdot \mathrm{mm}^{2}\right]$ & $10^{8}-10^{9}$ \\
\hline \hline Parameter & Identified value \\
\hline$k_{0}\left[\mathrm{~N} / \mathrm{mm}^{2}\right]$ & 0.012 \\
$k_{1}[-]$ & 1.91 \\
$E J\left[N \cdot \mathrm{mm}^{2}\right]$ & $4.66 \cdot 10^{8}$ \\
\hline
\end{tabular}

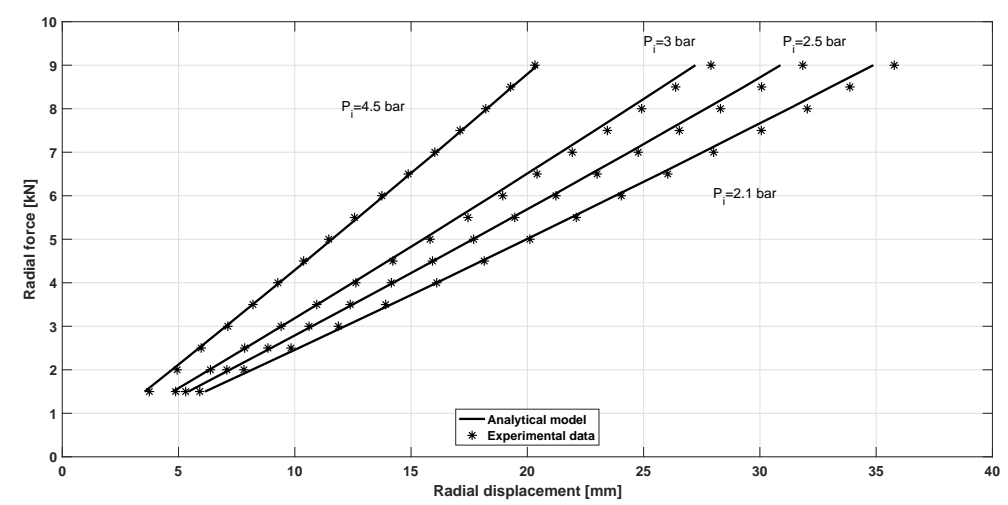

Fig. 11 Comparison between numerical and experimental data.

The FE model is experimentally validated by considering the force-displacement curves described in Section 3.

The FE model takes into account the actual shape and internal structure of the tire.

\subsection{Tire FE model}

The measured geometry of the tire reported in Fig. 10 has been discretized by finite elements. The actual structure of the tire has been modeled, i.e. the beads and the plies have been considered in the model. Fig. 12 shows the FEM model of the tire and the structural components are highlighted.

The rubber structure was divided in three main parts, namely the sidewall, the undertread and the tread. A Mooney-Rivlin model has been employed for describing the rubber material property.

Tyre reinforcements have been included and modelled by means of rebar layers embedded in the tire carcass. A 90 degree textile body ply was embedded in the whole cross section and a \pm 20 degree steel belt was added in the tire tread as shown in Fig. 12. 


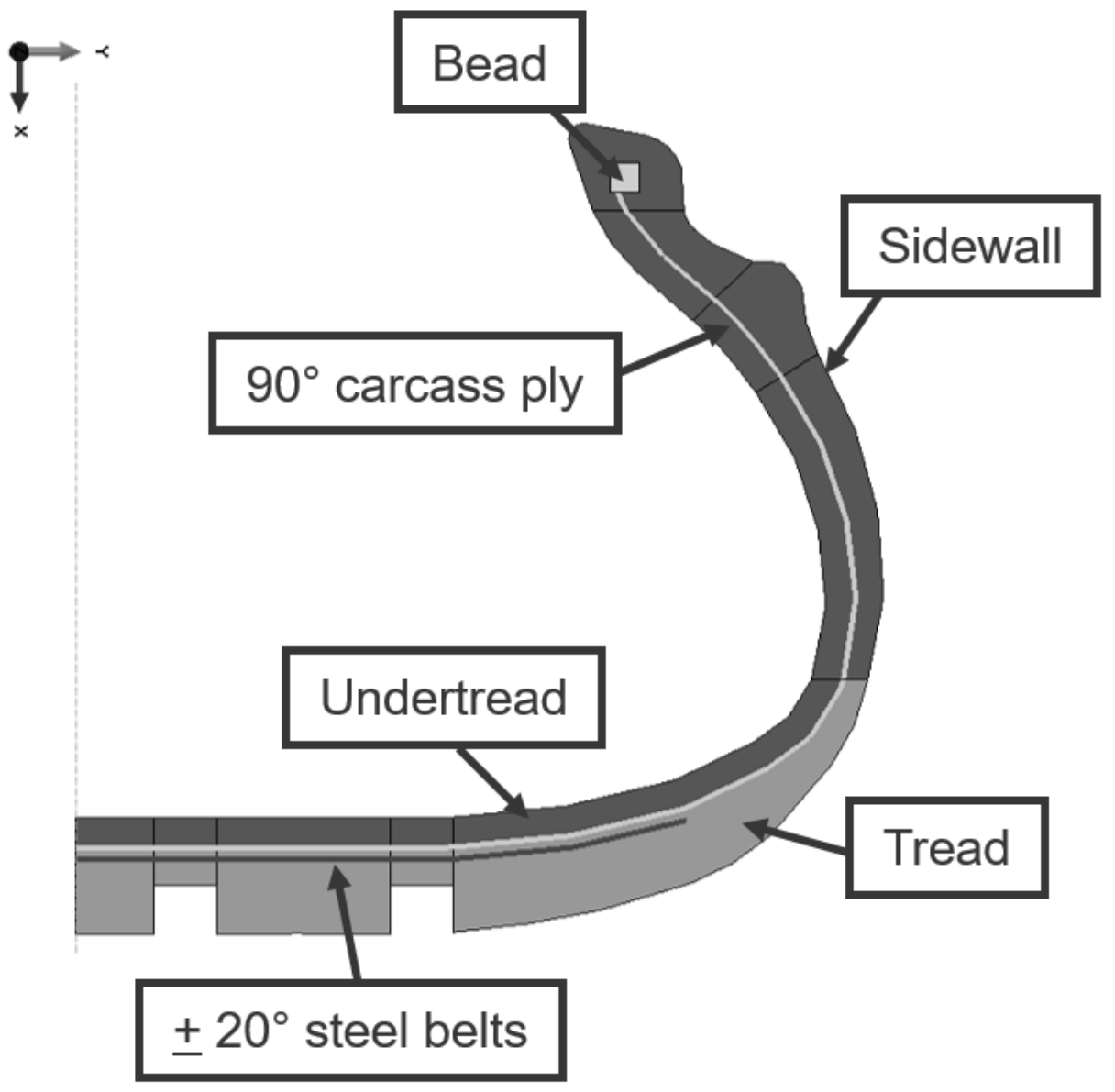

Fig. 12 Tire cross section, FEM model.

Table 4 and 5 summarize the material properties and the geometric properties of the reinforcements respectively.

The numerical simulation is divided in two steps [10-15, 33]:

$-2 \mathrm{D}$ axisymmetric FEM model of the inflated tire. The cross section of the tire is constrained at the rim interface and a uniform inflation pressure is applied at the inner surface as depicted in Fig. 13. During this step the position of the tire beads is adjusted to be in contact with the rim surface.

- 3D model generation by revolving the axisymmetric model and load application. In this step, the $2 \mathrm{D}$ axisymmetric model is revolved to generate the $3 \mathrm{D}$ model of the whole tire.

A mesh of linear brick elements is created for the 3D model, the average size of the mesh is $4 \mathrm{~mm}$.

A rigid plane that simulates the road surface has been included in the model. A frictional contact model has been employed for describing the interaction between the tire tread and the rigid plane. The 3D model of the tire is shown in Fig. 14. 
Table 4 Material properties of the tire structure. Taken from [12, 32].

\begin{tabular}{lll}
\hline & Material type & Value \\
\hline Bead & Isotropic & $E=210 \mathrm{GPa}, \nu=0.3$ \\
0 degree ply & Isotropic & $E=210 \mathrm{GPa}, \nu=0.3$ \\
90 degree ply & Isotropic & $E=5 \mathrm{GPa}, \nu=0.4$ \\
Sidewall and Undertread & Mooney-Rivlin & $C_{10}=0.14 \mathrm{MPa}, C_{01}=0.43 \mathrm{MPa}$ \\
Tread & Mooney-Rivlin & $C_{10}=0.80 \mathrm{MPa}, C_{01}=1.80 \mathrm{MPa}$ \\
\hline
\end{tabular}

Table 5 Geometric properties of reinforcements. Adapted from [12, 32].

\begin{tabular}{lll}
\hline & Distance between wires & Area per wire \\
\hline \pm 20 degree ply & $1 \mathrm{~mm}$ & $0.20 \mathrm{~mm}^{2}$ \\
90 degree body ply & $1 \mathrm{~mm}$ & $0.40 \mathrm{~mm}^{2}$ \\
\hline
\end{tabular}

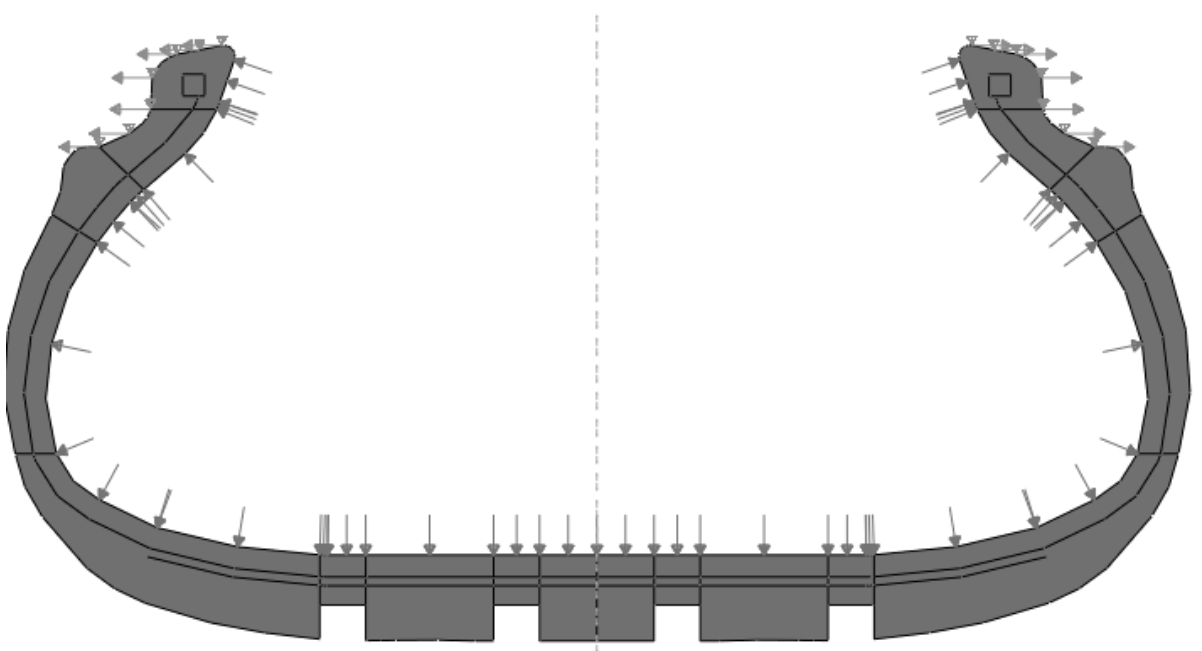

Fig. 13 Tire cross section subjected to inflation pressure - 2D axisymmetric model

The displacement of the rigid surface has been controlled and vertical and lateral stiffness tests have been simulated. In Fig. 15, the comparison between the radial and lateral forces computed by the $\mathrm{FE}$ model and the experimental data is shown for four different inflation pressures.

\subsection{Rim reaction forces}

In Fig. 16 reaction forces acting on the rim calculated by the analytical model are compared with the ones obtained from the FE model for two different inflation pressures (2.5 and 4.5 bar). The agreement between the reaction forces computed by the two models is quite good. 


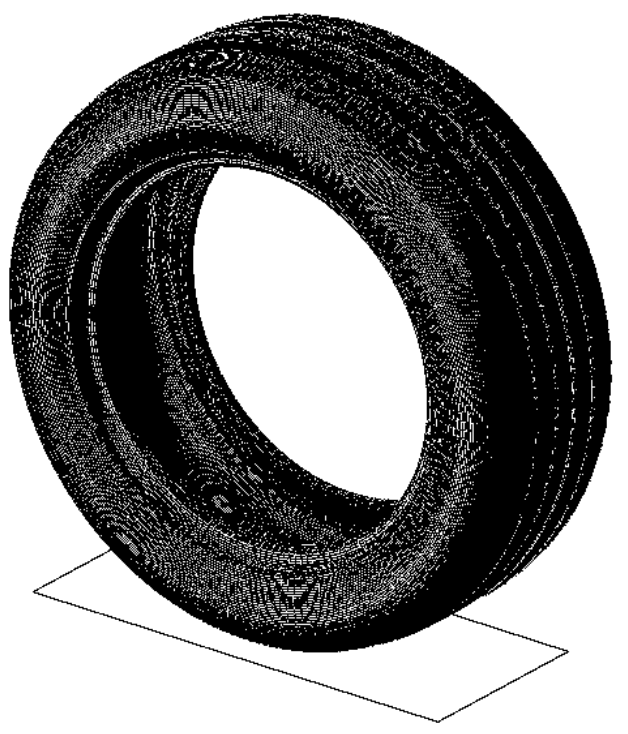

Fig. 14 Three-dimensional tire model

The analytical model is able to give a good approximation of the forces, while requiring a very limited set of information about the tire and a limited computational effort.

\section{Rim stress distribution}

In this section the stress field acting on the wheel rim has been analysed. The strain field at some critical location of the wheel rim has been measured by means of resistive strain gauges.

The measured strain field has been compared with the simulated one.

In the following section the experimental measurements are firstly described, then the different numerical models for computing the stress distribution on the wheel are presented and results are compared.

\subsection{Measure of the strain field on the wheel}

The wheel has been instrumented with a set of resistive strain gauges located at critical areas.

A series of 120 Ohm uniaxial strain gauges have been placed on the front and back sides of a spoke of the wheel as shown in Fig. 17. A triaxial strain rosette has been located near the connection fillet of the spoke and the central hub (S.G. A,B,C in Fig. 17).

Uniaxial strain gauges have been placed also on the wheel rim as shown in Fig. 18. The strain gauges are equally spaced by an angle of 9 degrees covering a sector of 36 degrees of the wheel rim. 

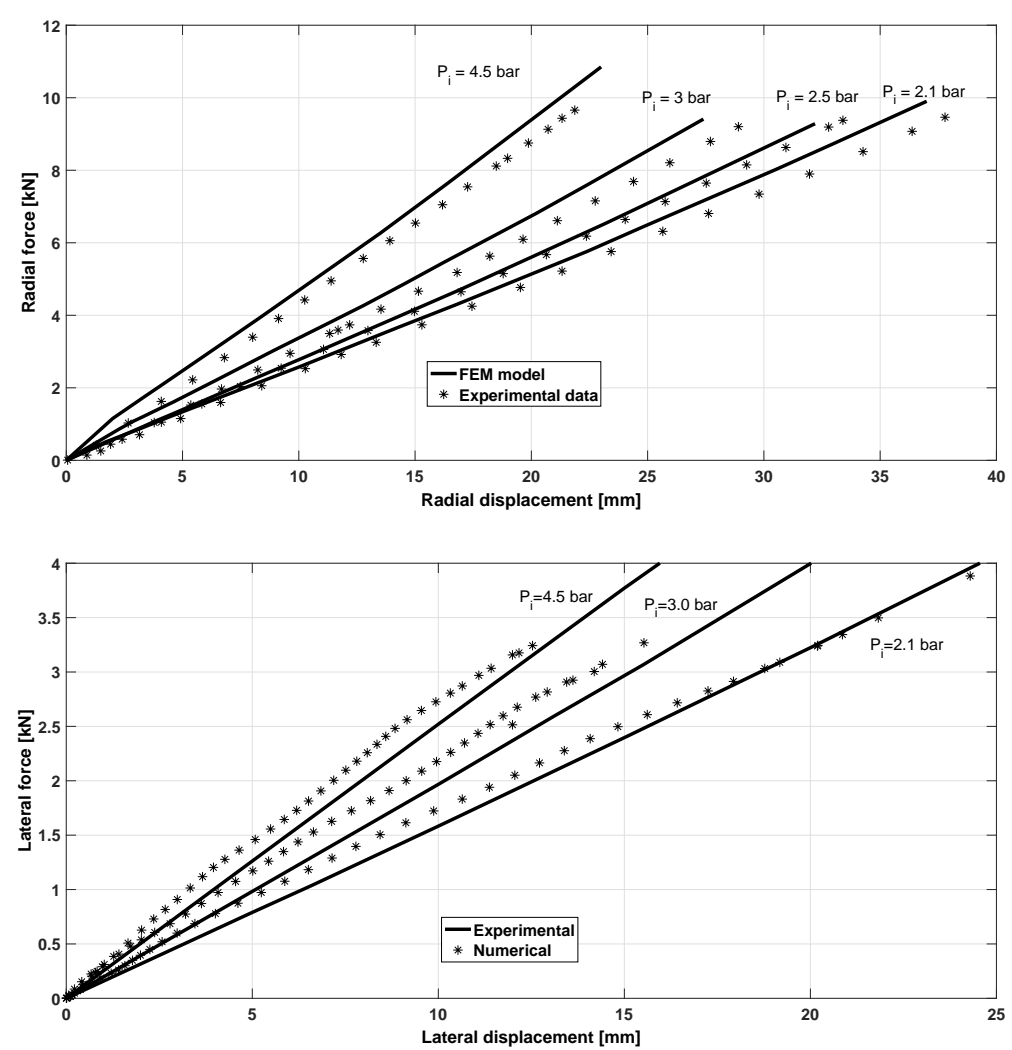

Fig. 15 Comparison between FE model results and experimental data. Top: radial forces (inflation pressures: 2.1, 2.5, 3 and 4.5 bar). Bottom: lateral forces (inflation pressures: 2.1, 3 and 4.5 bar).

The wheel has been fixed to the test bench and loaded as described in Sect. 3.1 .

\subsection{Numerical computation of the strain field}

The experimental test has been simulated by means of a FE model of the wheel. The wheel has been discretized with a second order tetrahedral mesh and constrained at the hub rear surface. The forces acting at tire rim interface due to a radial force acting at the contact patch have been modelled with four different approaches, namely cosine loading function, semi-analytical loading function, FEM loading function and complete model loading function.

\subsubsection{Cosine loading function}

The force distribution at the tire-rim interface is described by a cosine-distribution pressure acting at the bead seats $[3,6,7]$ as depicted in Fig. 19. The cosinedistribution pressure is described by the following expression 

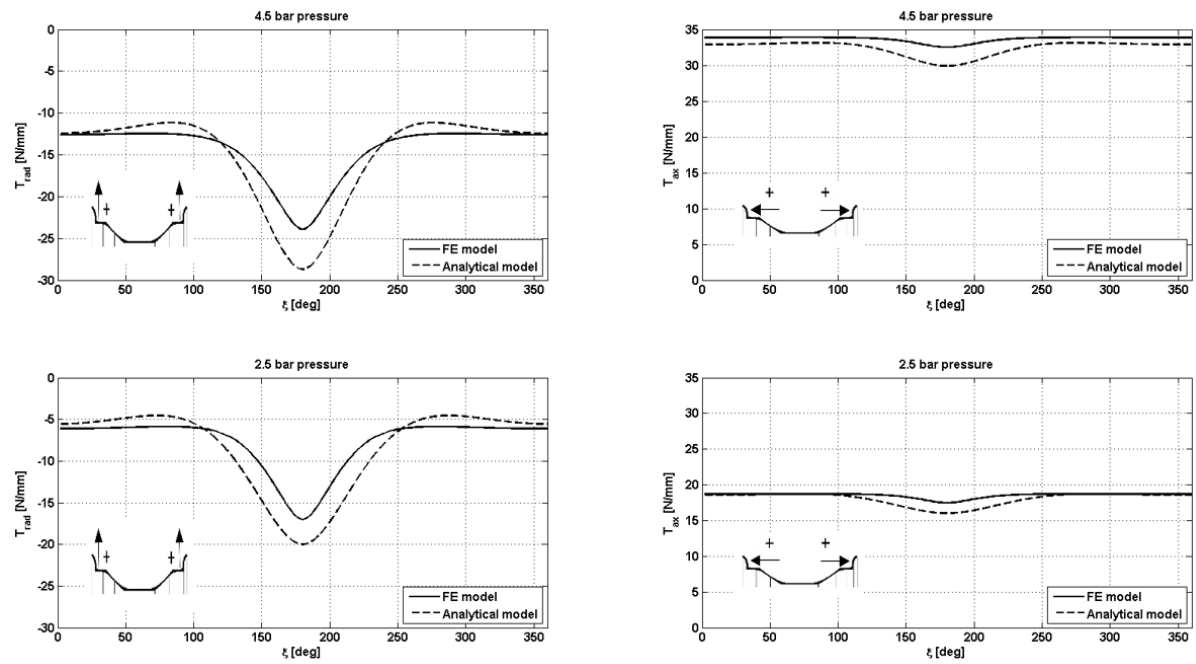

Fig. 16 Reaction forces on the rim (Fig. 6) computed by the FE model and by the analytical model respectively, for two inflating pressures (2.5 and 4.5 bar), radial load $8750 \mathrm{~N}$
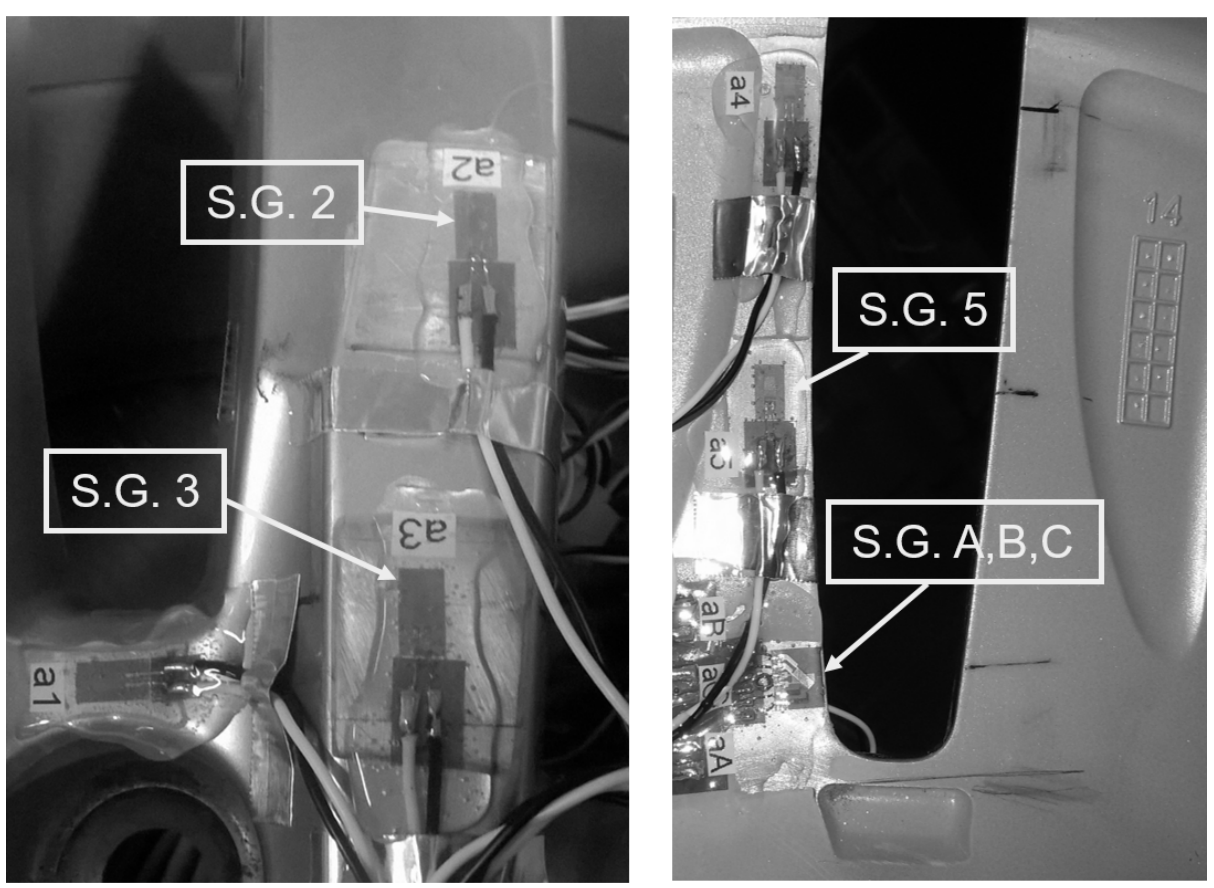

Fig. 17 Resistive strain gauges located on the front (left) and back (right) side of the spoke. 


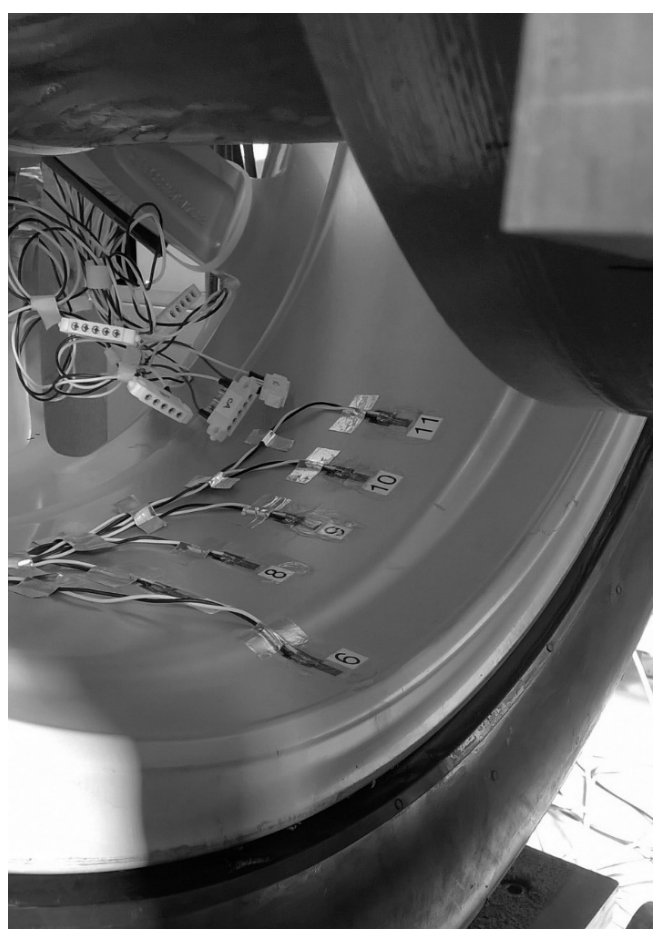

Fig. 18 Resistive strain gauges located on the wheel rim.

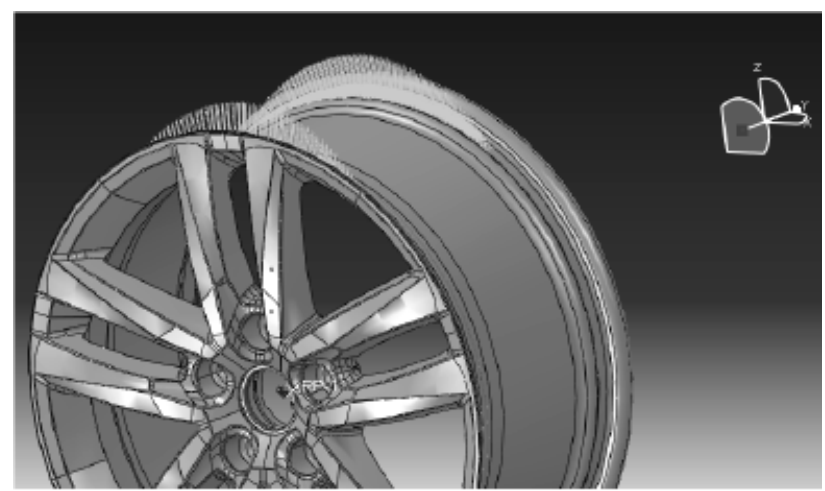

Fig. 19 Cosine-distribution pressure for applying vertical load.

$$
W_{r}=W_{0} \cos \left(\frac{\pi \gamma}{2 \gamma_{0}}\right)
$$

where $W_{r}$ is the distributed force, $W_{0}$ is the amplitude of the force distribution, $\gamma$ is the angular coordinate of a cylindrical reference system and $\gamma_{0}$ is the loading arch, that can be estimated from the contact patch length (eq. 30).

A uniform pressure distribution is added on the external surface of the wheel rim to account for the tire inflation pressure. Axial forces acting at the tire/rim 


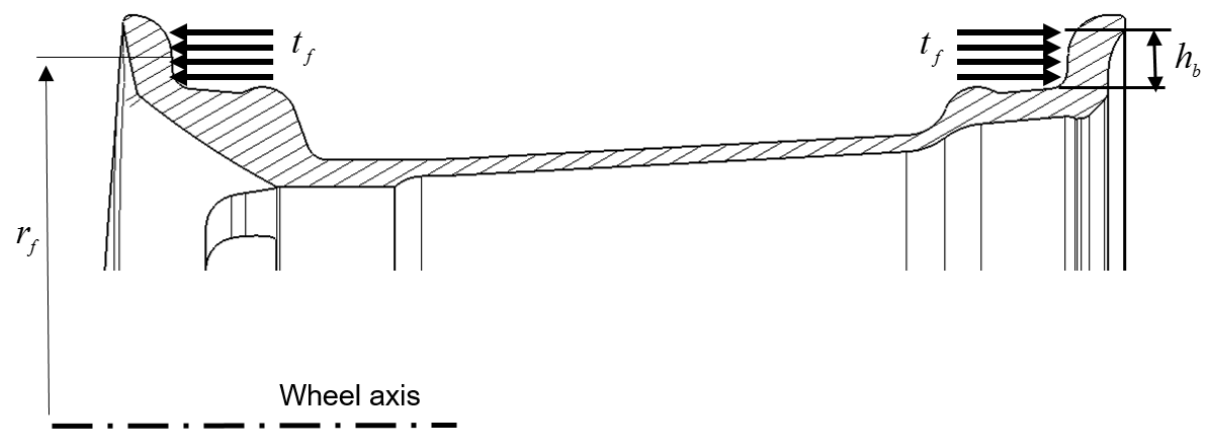

Fig. 20 Axial forces acting at the tire/rim interface.

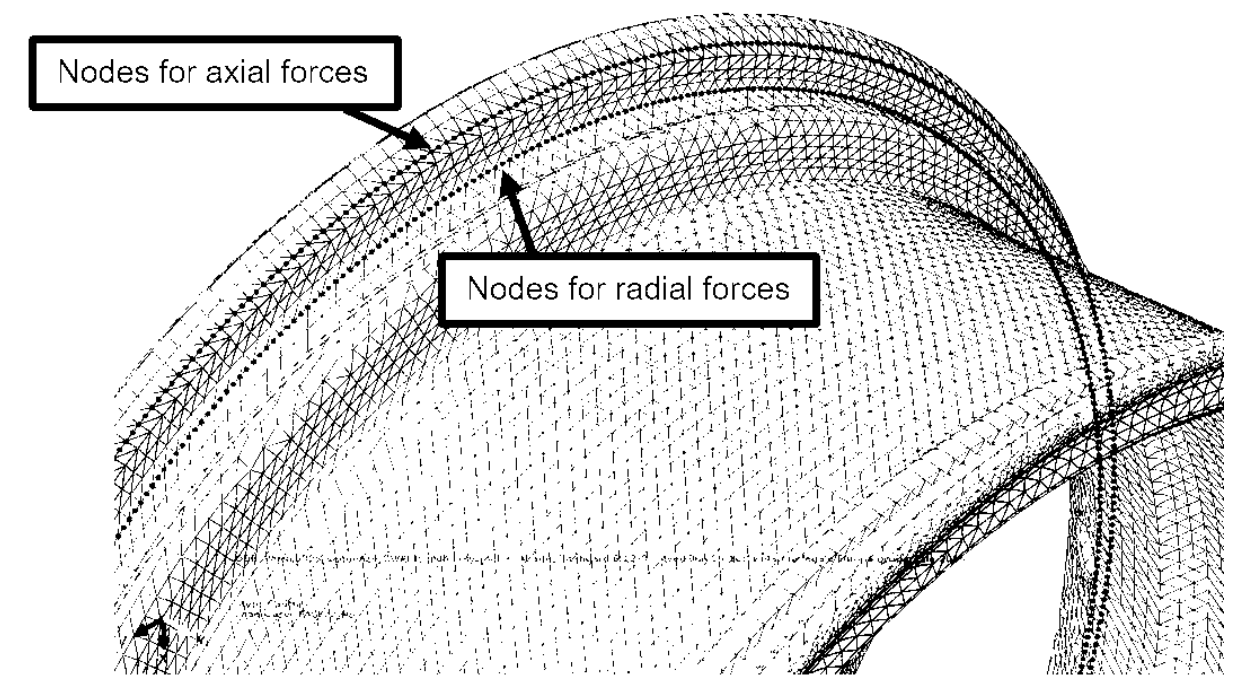

Fig. 21 Node sets for applying radial and axial reactions.

interface are introduced through the uniformly distributed pressure $t_{f}$ of Fig. 20 that acts on the whole rim flange. Being $P_{i}, R, h_{b}$ and $r_{f}$ the inflation pressure, the tire radius, the vertical surface of the rim flanges and the rim radius respectively (see Fig. 20), the expression of $t_{f}$ reads

$$
t_{f}=\frac{P_{i}}{4 r_{f} h_{b}}\left(R^{2}-r_{f}^{2}\right)
$$

\subsubsection{Semi-analytical loading function}

Reaction forces acting at the tire/rim interface computed with the semi-analytical model presented in section 2 and in Fig. 16 are introduced as nodal forces acting at the locations depicted in Fig. 21. A uniform pressure distribution is added on the external surface of the wheel rim to account for the tire inflation pressure. 


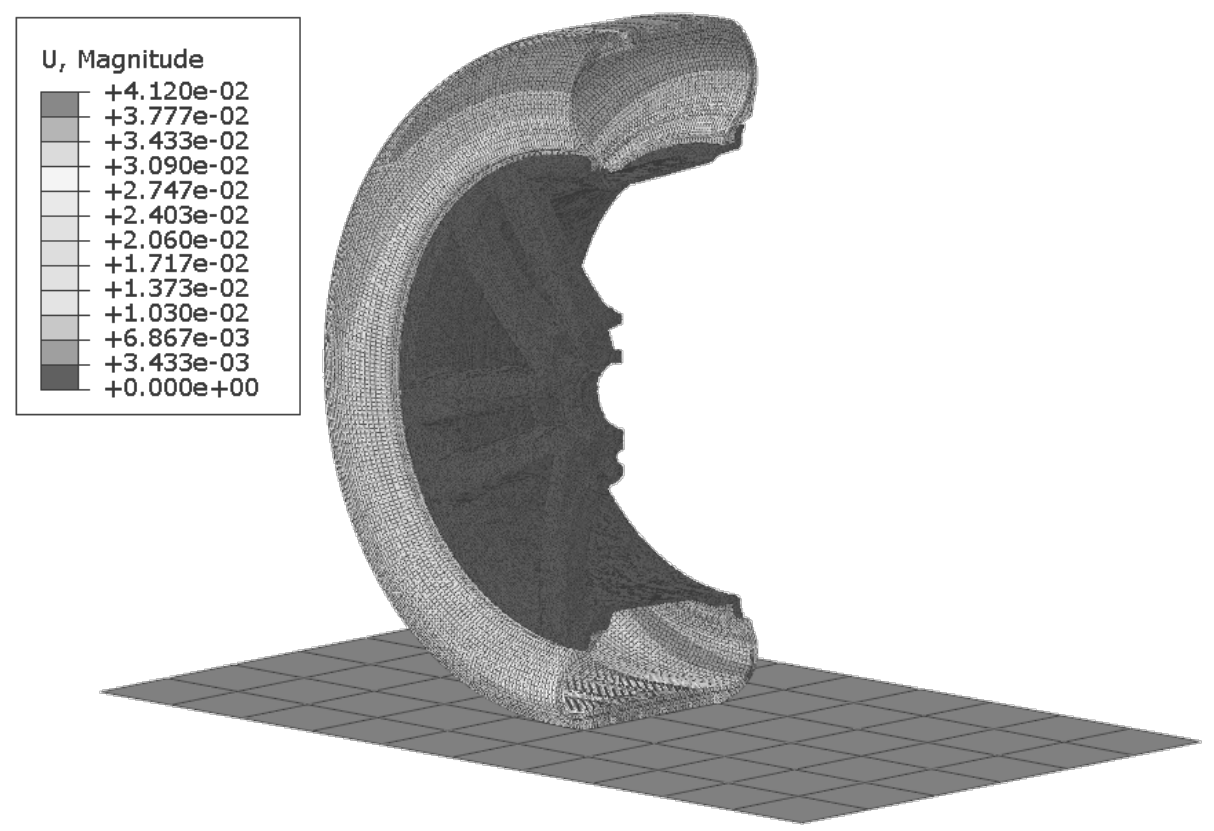

Fig. 22 Complete FE model, deformed configuration.

\subsubsection{FEM loading function}

The model is identical to the one considered in Section 5.2.2. In this case the forces at the tire-rim interface are obtained from the tire FE model described in Section 4 .

\subsubsection{Complete loading function}

The complete FE model is shown in Fig. 22. The physical model of the tire described in Section 4 and the wheel rim are considered. The interaction between the two parts is modelled through a frictional contact at the interface surface. A friction coefficient $\mu$ equal to 0.5 [34] has been considered for modelling the contact between the aluminium surface of the wheel rim and the rubber.

The symmetry of the problem has been exploited so that only half of the structure has been considered. The tire/ground contact forces have been applied through a rigid plane acting against the tire tread. The wheel is constrained at the central hub. A boundary condition of symmetry with respect to the mid transversal plane has been included in the FE model.

\subsection{Results and comparison}

Two different loading conditions have been tested:

- Tyre inflated at 4.5 bar and vertical load equal to $12990 \mathrm{~N}$

- Tyre inflated at 2.5 bar and vertical load equal to $8750 \mathrm{~N}$ 
Comparison between the numerically computed and measured strain field is reported in Table 6 and Table 7 for all the considered models.

Table 6 Comparison between computed and measured strain $\left[\frac{\mu m}{m}\right]$ - inflation pressure 4.5 bar, vertical load $12990 \mathrm{~N}$.

\begin{tabular}{llllll}
\hline & $\begin{array}{l}\text { Cosine loading } \\
\text { function }\end{array}$ & $\begin{array}{l}\text { Semi-analytical } \\
\text { loading } \\
\text { function }\end{array}$ & $\begin{array}{l}\text { FEM loading } \\
\text { function }\end{array}$ & $\begin{array}{l}\text { Complete } \\
\text { model loading } \\
\text { function }\end{array}$ & Experimental \\
\hline S.G. 2 & 385 & 396 & 319 & 218 & 283 \\
S.G. 3 & 267 & 292 & 231 & 302 & 212 \\
S.G. A & -549 & -522 & -440 & -433 & -410 \\
S.G. B & -228 & -234 & -206 & -206 & -166 \\
S.G. C & 127 & 126 & 108 & 97 & -422 \\
S.G. 5 & -568 & -552 & -462 & -454 & 415 \\
S.G. 7 & 684 & 404 & 413 & 419 & 400 \\
S.G. 8 & 657 & 396 & 404 & 410 & 367 \\
S.G. 9 & 587 & 374 & 381 & 387 & 340 \\
S.G. 10 & 497 & 344 & 351 & 358 & 310 \\
S.G. 11 & 419 & 308 & 320 & & 326 \\
\hline
\end{tabular}

Table 7 Comparison between computed and measured strain $\left[\frac{\mu m}{m}\right]$ - inflation pressure 2.5 bar, vertical load $8750 \mathrm{~N}$

\begin{tabular}{llllll}
\hline & $\begin{array}{l}\text { Cosine loading } \\
\text { function }\end{array}$ & $\begin{array}{l}\text { Semi-analytical } \\
\text { loading } \\
\text { function }\end{array}$ & $\begin{array}{l}\text { FEM loading } \\
\text { function }\end{array}$ & $\begin{array}{l}\text { Complete } \\
\text { model loading } \\
\text { function }\end{array}$ & Experimental \\
\hline S.G. 2 & 296 & 249 & 233 & 208 & 206 \\
S.G. 3 & 225 & 187 & 175 & 154 & 161 \\
S.G. A & -359 & -325 & -307 & -291 & -279 \\
S.G. B & -108 & -100 & -95 & -41 & -113 \\
S.G. C & 101 & 78 & 74 & 69 & -286 \\
S.G. 5 & -383 & -339 & -320 & -300 & 247 \\
S.G. 7 & 282 & 220 & 240 & 244 & 235 \\
S.G. 8 & 272 & 216 & 234 & 238 & 216 \\
S.G. 9 & 244 & 205 & 216 & 200 & 195 \\
S.G. 10 & 204 & 191 & 194 & 177 & 174 \\
S.G. 11 & 163 & 173 & 171 & & \\
\hline
\end{tabular}

Results of Table 6 and Table 7 show that the FEM reactions and complete model provide the best approximation of experimental data. The cosine loading function and the semi-analytical reactions overestimate the local deformation. The error of the cosine loading model is quite high for the strain gauges located on the wheel rim. The semi-analytical reactions model provides a better accuracy than the cosine loading function, especially if strain gauges on the wheel rim are considered. 


\section{Conclusions}

The paper has been devoted to the analysis of the interaction between tire and rim. In particular, the forces at the tire-rim interface have been studied by means of a simplified semi-analytical tire model and finite element analysis.

The semi-analytical tire model takes into account tread belt, sidewalls and pressure effects on the load carrying mechanism of the tire. The tread belt is modeled as a flexible ring connected to a rigid wheel rim by an elastic foundation. The elastic properties of the foundation consider two main contributions. The first contribution is given by the stiffness due to the inflating pressure and the geometrical variation of the tire section when loaded. The second contribution is given by the structural stiffness of the sidewalls modeled as curved beams. As a result, the stiffness of the elastic foundation connecting the belt to the rim is function of the inflating pressure and of the structural properties of the tire. The obtained model requires a limited set of data for its calibration, allowing its application even if the actual structure of the tire is not known into details. A simple experimental procedure is proposed for the calibration of the model.

A FE model, taking into account the actual structure of the tire, has been realized and used to compare the rim forces distribution computed by the semianalytical model. The force distributions obtained by the semi-analytical model show a quite good agreement with the distributions computed by the FE model. The curve shapes are very similar and a maximum error of the order of $20 \%$ has been found in the contact region.

Finally, the force distribution computed by the semi-analytical model has been employed for the computation of the stress in some significant locations of the wheel. The stresses computed in these location have been compared with the measurements obtained by strain gauges on the actual wheel. The stresses computed by using the force distribution obtained by the semi-analytical model have also been compared with stresses computed by using either a simpler force distribution (namely a cosine force distribution), either the force distribution obtained by the FE model of the tire, either a complete FE model considering both tire and wheel. The semi-analytical model has shown a good agreement with the experimental data. In particular, the semi-analytical model provides a very good accuracy (errors always below 5\%) if the strain gauges on the wheel rim are considered. With respect to the other models, it showed better performances with respect to the cosine distribution and slightly worse with respect to the two FE models.

In conclusion, despite its simplicity, the semi-analytical model has proved to be effective in describing the interaction between tire and rim and could be a useful tool especially when the structure of the tire is not known and the main interest is the way in which forces are transmitted between tire and wheel. Future developments can refer to the investigation of the effect of longitudinal force and camber.

\section{References}

1. H. Pacejka, Tyre and vehicle dynamics, Third edition. ButterworthHeinemann, Oxford., 2012. 
2. G. Mastinu and M. Ploechl, Road and Off-Road Vehicle System Dynamics Handbook. CRC Press, Boca Raton., 2014.

3. J. Stearns, T. Srivatsan, X. Gao, and P. Lam, "Understanding the influence of pressure and radial loads on stress and displacement response of a rotating body: The automobile wheel," International Journal of Rotating Machinery, vol. 2006, pp. 1-8, 2006.

4. S. Das, "Design and weight optimization of aluminum alloy wheel," International Journal of Scientific and Research Publications, vol. 4, pp. 1-12, 2014.

5. L. Wang, Y. Chen, C. Wang, and Q. Wang, "Fatigue life analysis of aluminium wheels by simulation of rotary fatigue test," Journal of Mechanical Engineering, vol. 57, pp. 31-39, 2011.

6. P. R. Raju, B. Satyanarayana, K. Ramji, and K. S. Babu, "Evaluation of fatigue life of aluminium alloy wheels under radial loads," Engineering Failure Analysis, vol. 14, pp. 791-800, 2007.

7. J. Stearns, T. Srivatsan, A. Prakash, and P. Lam, "Modeling the mechanical response of an aluminium alloy automotive rim," Materials Science and Engineering, vol. A366, pp. 262-268, 2004.

8. W. Hall, J. Mottram, and R. Jones, "Tire modeling methodology with the explicit finite element code ls-dyna," Tire Science and Technology, vol. TSTCA 32, pp. 236-261, 2004.

9. G. Meschke, H. Prayer, and H. Mang, " $3 \mathrm{~d}$ simulations of automobile tires, mnesh generation and solution strategies: material modeling," Tire Science and Technology, vol. TSTCA 25, pp. 154-176, 1997.

10. X. Yang, Finite element analysis and experimental investigation of tyre characteristics for developing strain-based intelligent tyre systems. Doctoral dissertation, University of Birmingham, 2011.

11. X. Yan, "Non-linear three-dimensional finite element modeling of radial tires," Mathematics and Computers in Simulation, vol. 58, pp. 51-70, 2001.

12. E. Tönük and Y. Ünlüsoy, "Prediction of automobile tire cornering force characteristics by finite element modeling and analysis," Computers and Structures, vol. 79, pp. 1219-1232, 2001.

13. N. Korunović, M. Trajanović, and M. Stojković, "Fea tires subjected to static loading," Journal of the Serbian Society for Computational Mechanics, vol. 1, pp. 87-98, 2007.

14. M. Ghoreisky, "Finite element analysis of the steel-belted radial tyre with tread pattern under contact load," Iranian Polymer Journal, vol. 15, pp. 667-674, 2006.

15. N. Korunović, M. Trajanović, M. Stojković, D. M. ` sić, and J. Milovanović, "Finite element analysis of a tire steady rolling on the drum and comparison with experiment," Journal of Mechanical Engineering, vol. 57, pp. 888-897, 2011.

16. G. Mastinu, S. Gaiazzi, F. Montanaro, and D. Pirola, "A semi-analytical tyre model for steady and transient-state simulations," Vehicle System Dynamics, vol. 27, pp. 2-21, 1997.

17. G. Mastinu, G. Previati, and M. Gobbi, "Analytical computation of the radial stiffness of pneumatic tyres," in 22nd IAVSD Symposium on Dynamics of Vehicles on Roads and Tracks, (Manchester, UK), 2011.

18. T. Rhyne, "Development of a vertical stiffness relationship for belted radial tires," Tire Science and Technology, vol. 33, pp. 136-155, 2005. 
19. J. Pauwelussen, L. Gootjes, C. Schröder, K.-U. Köhne, S. Jansen, and A. Schmeitz, "Full vehicle abs braking using the swift rigid ring tyre model," Control Engineering Practice, vol. 11, pp. 199-207, 2003.

20. S. Jansen, P. Zegelaar, and H. Pacejka, "The influence of in-plane tyre dynamics on abs braking of a quarter vehicle model," Vehicle System Dynamics, vol. 32, pp. 249-261, 1999.

21. S. Kim, P. Nikravesh, and G. Gim, "A two-dimensional tire model on uneven roads for vehicle dynamic simulation," Vehicle System Dynamics, vol. 46, pp. 913-930, 2008.

22. P. Kindt, P. Sas, and W. Desmet, "Development and validation of a threedimensional ring-based structural tyre model," Journal of Sound and Vibration, vol. 326, pp. 852-869, 2009.

23. L. Kung, W. Soedel, and T. Yang, "Free vibration of a pneumatic tire-wheel unit using a ring on an elastic foundation and a finite element model," Journal of Sound and Vibration, vol. 107, pp. 181-194, 1986.

24. F. Ballo, M. Gobbi, G. Mastinu, G. Previati, and R. Zerboni, "Motorcycle tire modeling," in Proc. of the ASME 2015 IDETC/CIE 2015, (Boston, Massachussetts, USA), 2015.

25. G. Gobbi, F. Ballo, G. Mastinu, and G. Previati, "Motorcycle tire modeling for the study of tire-rim interaction," Journal of Mechanical Design, vol. 138, pp. 051404-051404-13, 2016.

26. W. Young and R. Budynas, Roark's Formulas for Stress and Strain. $7^{\text {th }}$ edition. McGraw-Hill Professional, New York U.S.A., 2001.

27. G. Mastinu and M. Fainello, "Study of the pneumatic tyre behaviour on dry and rigid road by finite element method," Vehicle System Dynamics, vol. 21, pp. 143-165, 1992.

28. G. Mastinu, M. Gobbi, and G. Previati, "A new six-axis load cell. part i: Design," Experimental Mechanics, vol. 51, pp. 373-388, 2011.

29. M. Gobbi, G. Previati, P. Guarnieri, and G. Mastinu, "A new six-axis load cell. part ii: Error analysis, construction and experimental assessment of performances," Experimental Mechanics, vol. 51, pp. 389-399, 2011.

30. F. Ballo, M. Gobbi, G. Mastinu, and G. Previati, "Advances in force and moments measurements by an innovative six-axis load cell," Experimental $\mathrm{Me}$ chanics, vol. 54, pp. 571-592, 2014.

31. G. Holzapfel, Nonlinear Solid Mechanics: A continuum Approach for Engineering. Wiley, 2000.

32. V. Alkan, S. Karamihas, and G. Anlas, "Finite element modeling of static tire enveloping characteristics," International Journal of Automotive Technology, vol. 12 , pp. 529-535, 2011.

33. G. Previati and M. Kaliske, "Crack propagation in pneumatic tires: Continuum mechanics and fracture mechanics approaches," International Journal of Fatigue, vol. 37, pp. 69-78, 2012.

34. R. Pelle, "Fem simulation of the tire/rim seating process," Tire Science and Technology, vol. 22, pp. 76-98, 1994. 


\section{A Coefficients of the beam equations}

Under the boundary conitions describd in section 2.3 , the five constants of equation 28 read

$$
\left\{\begin{array}{l}
C_{0}=\frac{\operatorname{Pr}^{3}}{2 \pi E J \delta^{2}} \\
C_{1}=\frac{\operatorname{Pr}^{3}[\alpha \cosh (\alpha \pi) \sin (\beta \pi)+\beta \cos (\beta \pi) \sinh (\alpha \pi)]}{2 E J \alpha \beta\left(\alpha^{2}+\beta^{2}\right)[\cos (2 \pi \beta)-\cosh (2 \pi \alpha)]} \\
C_{2}=0 \\
C_{3}=0 \\
C_{4}=\frac{\operatorname{Pr}^{3}[\beta \cosh (\alpha \pi) \sin (\beta \pi)-\alpha \cos (\beta \pi) \sinh (\alpha \pi)]}{2 E J \alpha \beta\left(\alpha^{2}+\beta^{2}\right)[\cos (2 \pi \beta)-\cosh (2 \pi \alpha)]}
\end{array}\right.
$$

The described model has been derived considering a concentrated vertical force applied to the tire. In an actual tire, the vertical force is applied by means of a pressure distribution in the contact patch. In this case, the contact patch can be discretised in a number of intervals and the solution can be obtained by superimpositions of the effects (see eq. 29). 